\title{
Oxigenoterapia de alto flujo: ¿Todas las insuficiencias son iguales?
}

\author{
High flow nasal cannula oxygen therapy. \\ Are all respiratory failure the same?
}

Juan Higuera', David Cabestrero'

\section{ABSTRACT}

Introduction: High-flow nasal cannula is an oxygenation therapy in patients with acute respiratory failure. There are, some questions about this technique which answer is needed. Matherial and Methods: We analyzed all patients that require high-flow nasal cannula oxygen therapy admitted to a polyvalent intensive care unit of a university tertiary hospital. We select those patients who require the therapy as initial support for their acute respiratory failure. We analyzed the mortality and connection to mechanical ventilation (V.M.) rate. We performed a global analysis and then a sub analysis for underlying pathology. Our objective is to describe which pathology has the best results with this treatment. Patients who require therapy to support scheduled extubation, heart failure, pulmonary thromboembolism, thoracic trauma and decreased level of consciousness were excluded. Results: We analysed a total of 128 patients. 76 Men, Mean age 57.4 years, APACHE II 19; SOFA 8.2; SAPS II 55.2; Mean income 13.3 days; Patients required the therapy an average of 2.8 days. 65 patients required connection to M.V. after HFNC therapy. Those patients who were intubated in the first 48 hours had a mortality rate of $45 \%$ while those in which the therapy was delayed more than 48 hours had a mortality rate of $56 \%(P=0.3)$. Patients were divided according to their underline pathology. Acute respiratory failure: Extra pulmonary or intra pulmonary (Pneumonia in immunosuppressed and Pneumonia in immuno competent). In the first group, connection to MV was required in $54 \%$ of the cases, with a mortality rate of $54 \%$ in those intubated in the first 48 hours vs $40 \%$ later. In the group of pneumonia in immunosuppressed patients, M.V. was required in the $60.5 \%$ of the cases with a mortality rate of $75 \%$ in those intubated in the first 48 hours vs $71 \%$ posteriorly. In the group Pneumonia in immuno competent, M.V. was required in $42 \%$ of the cases with a mortality rate
\end{abstract}

\section{Key words:}

Acute respiratory failure, High flow nasal cannula oxygen therapy, Invasive mechanical ventilation,

Adults

Hospital Universitario Ramón y Cajal, España.

Fecha de ingreso: 23 de julio de 2018

Fecha de aprobación: 10 de agosto de 2018

ORCID

https://orcid.org/0000-0003-1778-2291

Correspondencia:

Juan Higuera

E-mail: jhiguera.151@gmail.com 
of $10 \%$ in the ones intubated the first 48 hours vs $50 \%$ later. Statistically significant differences were observed regarding the need of M.V connection according to base pathology. Conclusions: Results of HFNC oxygen therapy do not appear to be different in pulmonary or extrapulmonary respiratory failure. The severity of the patient ilness is related to the need of mechanical ventilation and mortality rate. The patient who benefits the most from the early identification of the failure of this therapy, is the one who presents acute respiratory failure due to pneumonia in immunocompetent patient.

\section{RESUMEN}

Introducción: La oxigenoterapia de alto flujo es una técnica de oxigenación en los pacientes con insuficiencia respiratoria aguda. Existen, algunas preguntas acerca de esta técnica que necesitan respuesta. Material y Métodos: Analizamos todos los pacientes que requieren oxigenoterapia de alto flujo ingresados en una Unidad de Cuidados Intensivos polivalente de un hospital terciario universitario. Seleccionamos aquellos pacientes que requieren la terapia como soporte inicial de su insuficiencia respiratoria aguda. Analizamos la mortalidad y conexión a ventilación mecánica (V.M.). Realizamos un análisis global y posteriormente un subanálisis por patología subyacente. Nuestro objetivo es describir en que patología presenta mejores resultados. Se excluyen los pacientes que requieren la terapia como apoyo a la extubación programada, insuficiencia cardiaca, tromboembolismo pulmonar, trauma torácico y disminución del nivel de conciencia. Resultados: Analizamos un total de 128 pacientes. Setenta y seis varones, edad media 57,4 años, APACHE II 19; SOFA 8,2; SAPS II 55,2; días de ingreso medio 13,3; Los pacientes requieren la terapia una media de 2,8 días. Sesenta y cinco pacientes requieren conexión a V.M. tras uso de esta terapia. Aquellos pacientes que son intubados en las primeras 48 horas presentan una mortalidad de $45 \%$, mientras que aquellos en los que la terapia se alarga más de 48 horas la mortalidad es del $56 \%(p=0,3)$. Se divide a los pacientes según su patología de base. Insuficiencia respiratoria de causa: Extrapulmonar, neumonía en inmunodeprimido y neumonía en inmuno competente. En el primer grupo se objetiva conexión a V.M en un $54 \%$ de los casos, con una mortalidad del $54 \%$ en aquellos intubados en las primeras 48 horas vs $40 \%$ posteriormente. En el grupo neumonía en inmunodeprimido se requiere V.M en el 60,5\% con una mortalidad del $75 \%$ en aquellos intubados en las primeras 48 horas vs $71 \%$ posteriormente. En el grupo neumonía en inmunocompetente objetivamos conexión a V.M. en el $42 \%$ con una mortalidad del $10 \%$ en los intubados las primeras 48 horas vs $50 \%$ posteriormente. Se objetivan diferencias estadísticamente significativas respecto a necesidad de conexión a V.M. según patología de base. Conclusiones: Los resultados de la terapia de alto flujo no parece ser diferente en la insuficiencia respiratoria de causa pulmonar o extrapulmonar. La gravedad del enfermo se relaciona con la necesidad de ventilación mecánica y mortalidad. El paciente que más se beneficia de la rápida identificación del fracaso de esta terapia es el que presenta insuficiencia respiratoria por neumonía en paciente inmunocompetente.

\section{Palabras clave:}

Insuficiencia Respiratoria Aguda, Oxigenoterapia de Alto Flujo, Ventilación Mecánica Invasiva, Adultos 


\section{Introducción}

a oxigenoterapia de alto flujo es una técnica de oxigenación en los pacientes con insuficiencia respiratoria aguda. Su uso se está incrementando a lo largo de los últimos años[1].

Esta terapia presenta ventajas frente a las técnicas de oxigenación convencional mediante mascarillas o de bajo flujo, aporta un flujo de oxígeno, sólo, o mezclado con aire, por encima del flujo pico inspiratorio del paciente a través de una mascarilla o cánula nasal, y alcanza flujos medios entre 7-60 litros por minuto. El gas se calienta hasta un valor cercano a la temperatura corporal $\left(34-40^{\circ}\right)$ y se humidifica (95-100\%; $44 \mathrm{mg} / \mathrm{L}_{2} \mathrm{O}$ ), siendo éste mejor tolerado[2],[3].

El procedimiento inunda el espacio nasofaríngeo con gas optimizando cada inspiración, genera una presión positiva en la vía aérea [4],[5],[6] y presenta la ventaja de mantener una fracción inspiratoria de oxígeno constante y estable, a diferencia de las técnicas antes mencionadas[7].

Su expansión, ha causado que haya preguntas que queden aún por resolver. Su principal indicación es la insuficiencia respiratoria aguda de causa hipoxémica. Esto, ha abierto nuevas preguntas como si su uso supera a las técnicas convencionales[8], si evita intubaciones[9], si evita reintubaciones en la extubación programada[10], o si el reconocimiento tardío de su fracaso aumenta la mortalidad[11].

Existen pocas referencias en la bibliografía actual sobre cómo identificar el fracaso de la oxigenoterapia de alto flujo y cuándo progresar a una intubación oro traqueal y conexión a la ventilación mecánica convencional[12], o si su éxito, fracaso o identificación dependen de la patología o gravedad subyacente $[13],[14],[15]$.

Nuestro objetivo es analizar los pacientes en los que se utiliza la oxigenoterapia de alto flujo como tratamiento de la insuficiencia respiratoria aguda en una Unidad de Cuidados Intensivos. Queremos responder a la pregunta de si la oxigenoterapia de alto flujo presenta menores índices de conexión a ventilación mecánica o mortalidad en la insuficiencia respiratoria de causa pulmonar primaria frente a la insuficiencia respiratoria secundaria a un proceso extrapulmonar, y si este proceso se ve influido si el paciente es o no inmunocompetente. Además, queremos describir el porcentaje de pacientes en los que se reconoce el fallo de la oxigenoterapia de alto flujo en las primeras 48 horas, analizando su mortalidad según patología subyacente.

\section{Material y Métodos}

Se realizó un estudio retrospectivo, observacional, con todos los enfermos que ingresaron en un Servicio de Medicina Intensiva de un Hospital Terciario y Universitario, y requirieron oxigenoterapia de alto flujo como tratamiento de la insuficiencia respiratoria aguda.

El estudio contó con la aprobación del Comité Ético de Investigación Clínica del Hospital Ramón y Cajal. El tratamiento de los datos fue realizado con las medidas de seguridad establecidas en cumplimiento de la Ley Orgánica 15/1999 de protección de datos de carácter personal.

Se recogieron todos los enfermos consecutivos en los que se utilizó la oxigenoterapia de alto flujo entre mayo de 2013 hasta abril de 2016. El estudio busca analizar los resultados en aquellos pacientes en los que se utilizó la oxigenoterapia de alto flujo como soporte inicial de su insuficiencia respiratoria aguda de causa parenquimatosa pulmonar primaria o secundaria.

La causa de la insuficiencia respiratoria aguda se definió como "hipoxémica" $\left(\mathrm{PO}_{2}<60\right.$ mmhg o saturación mediante pulsioximetría $<88 \%$ ), "hipercápnica" $\left(\mathrm{PCO}_{2}>45 \mathrm{mmhg}\right)$ o mixta.

"Causa primaria" se definió como la insuficiencia respiratoria por afectación pulmonar infecciosa directa como foco más probable por clínica, microbiología o patrón radiológico. La neumonía fue clasificada según la comorbilidad del enfermo, en "neumonía en paciente inmunocompetente" y "neumonía en paciente inmunodeprimido". Los pacientes se clasificaron como "inmunodeprimidos", si presentaban una enfermedad oncohematológica o contaban con antecedentes personales de trasplante de médula. La "causa secundaria" se definió como aquella insuficiencia respiratoria cuya etiología es una afectación pulmonar secundaria a un proceso inflamatorio o infeccioso sistémico.

Los pacientes definidos en el grupo "causa primaria" son aquellos que presentaron una insuficiencia respiratoria, de etiología infecciosa, cuyo foco primario más probable es el respiratorio. Los "pacientes inmunodeprimidos" son aquellos que presentaron enfermedades hematológicas malignas o se encontraban bajo un tratamiento inmunosupresor. Aquellos pacientes clasificados como "causa secundaria", se definieron como pacientes que desarrollaron una insuficiencia respiratoria de forma secundaria a una reacción inflamatoria primaria extrapulmonar.

En nuestro grupo de enfermos, se inició la oxigenoterapia de alto flujo a 40 litros (20 aire y 20 de $\mathrm{O}_{2^{\prime}}$ 
correspondiente a una $\mathrm{FiO}_{2}$ de 0,6). El objetivo de la oxigenación con la terapia de alto flujo, fue mantener una saturación de $\mathrm{O}_{2}$ por pulsioximetría mayor del $88 \%$. Cuando la saturación de $\mathrm{O}_{2}$ disminuía por debajo de esta cifra, se aumentaron los litros por minuto a 50 litros con $\mathrm{FiO}_{2}$ del $80-90 \%$. Cuando el paciente precisaba mayores requerimientos de $\mathrm{FiO}_{2} \mathrm{O}$ aumentaba su trabajo respiratorio, se consideraba fallo de oxigenoterapia de alto flujo y se progresaba a ventilación mecánica invasiva. Ningún paciente recibió ventilación mecánica no invasiva.

Se consideró "fallo de la oxigenoterapia de alto flujo", cuando el paciente recibió ventilación mecánica invasiva, como consecuencia de la imposibilidad de poder oxigenarse adecuadamente, aplicando los criterios anteriormente especificados. Otra causa de fracaso y requerimiento de ventilación mecánica fueron el desarrollo de encefalopatía hipercápnica, coma, parada cardiorrespiratoria, situación de shock de cualquier etiología o la mala tolerabilidad clínica. Se consideró "fracaso tardío" como aquellos pacientes que se mantuvieron con oxigenoterapia de alto flujo durante más de 48 horas, pero que no presentaron una mejoría en la oxigenación respecto a su situación basal.
Se analizaron las variables: Edad, sexo, índices de gravedad: SOFA, APACHE II, SAPS II, diagnóstico tabulado, causa de la insuficiencia respiratoria, días de ingreso en medicina intensiva, días de uso del alto flujo antes de la intubación, porcentaje de pacientes que requieren ventilación mecánica y mortalidad durante su estancia en Medicina Intensiva.

Se han analizado los pacientes que requieren ventilación mecánica tras el fracaso de la oxigenoterapia de alto flujo. El porcentaje de mortalidad en aquellos en los que el fracaso se identifica en las primeras 48 horas y son conectados a ventilación mecánica, y aquellos en los que la conexión se produce después de 48 horas.

Se incluyeron 174 enfermos inicialmente, todos aquellos que habían ingresado en la Unidad de Cuidados Intensivos y habían requerido oxigenoterapia de alto flujo en algún momento de su evolución. Se excluyeron todos los pacientes que requirieron la terapia de alto flujo, como apoyo a la extubación programada, y aquellos en los que se utilizó el alto flujo como tratamiento de la insuficiencia respiratoria cuya causa no es parenquimatosa pulmonar, primaria o secundaria.

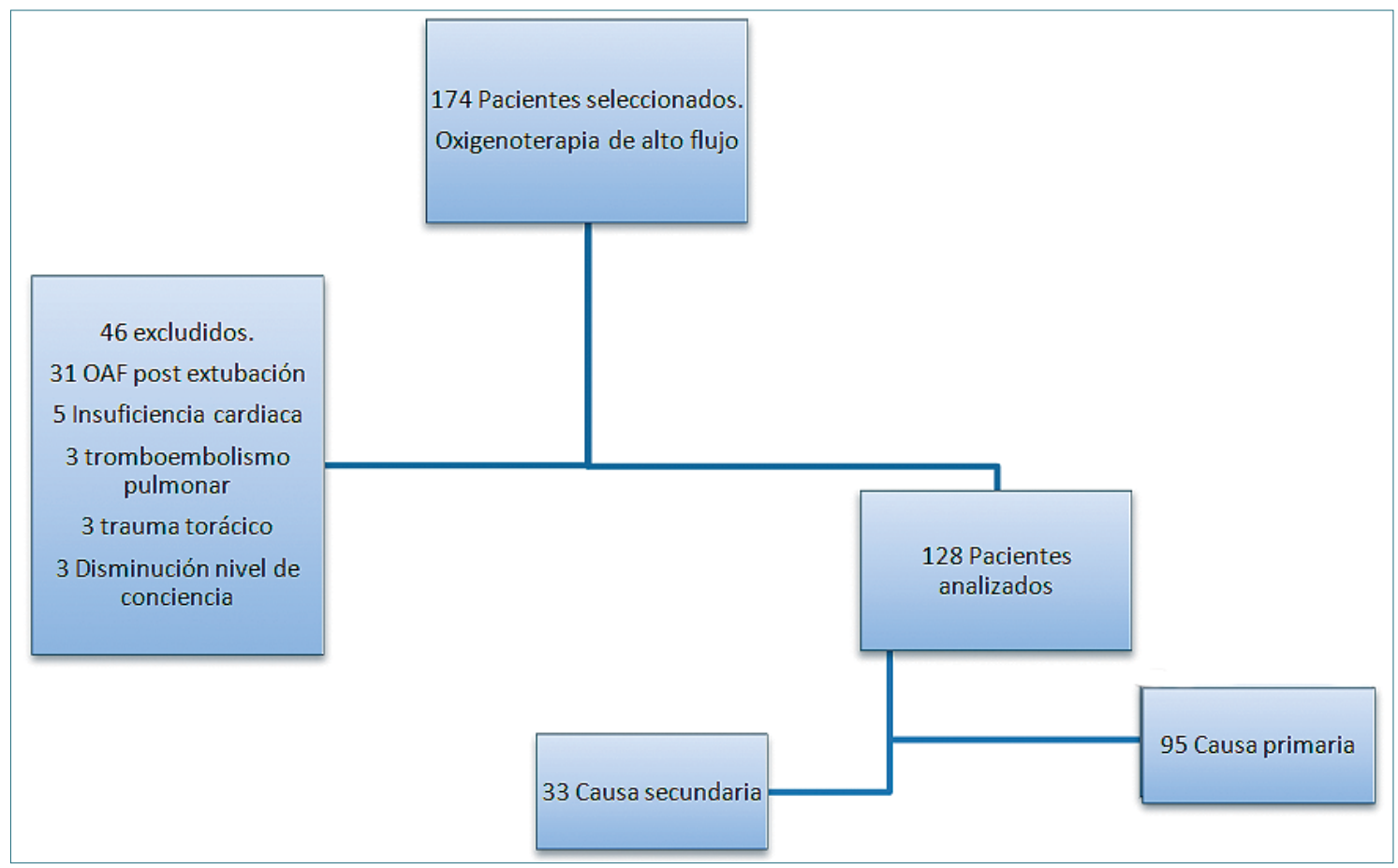

Figura 1. En la figura se muestra la selección de pacientes. Inicialmente se seleccionan 174 (todos los que ingresan en UCI y requieren terapia de alto flujo). Cuarenta y seis excluidos por las características señaladas y los 128 analizados en los que se utiliza la oxigenoterapia de alto flujo como tratamiento inicial de la insuficiencia respiratoria con las características señaladas. 
Los pacientes excluidos del estudio fueron pacientes cuya causa de insuficiencia respiratoria fueron: tromboembolismo pulmonar (4), edema agudo de pulmón (cardiogénico) (5), alteración del nivel de conciencia (3), trauma torácico (3) o uso de oxigenoterapia de alto flujo como apoyo a la extubación programada exclusivamente (31). Se excluyeron un total de 46 pacientes (Figura 1).

Se realiza el análisis estadístico mediante la herramienta IBM ${ }^{\circledR}$ SPSS Statistics 23 . La normalidad de las variables ha sido establecida mediante el test de Komogorov-Smirnov. Las variables cuantitativas con distribución normal han sido expresadas como media \pm desviación estándar (rango). Estas, han sido comparadas mediante test $\mathrm{t}$-Student o ANOVA. Las distribuciones cuantitativas que no seguían una distribución normal han sido comparadas utilizando test de Wilcoxon, y son expresadas como mediana y rango intercuartílico. Las variables cualitativas son mostradas como números y porcentajes. Son comparadas mediante test de McNemar y Chi-cuadrado. El nivel de significación estadística se ha establecido con valores de $\mathrm{P}$ menores de 0,05 . Los resultados son expresados como intervalos de confianza del 95\%.

\section{Resultados}

Se recogieron un total de 128 pacientes. Varones $76(59,4 \%)$, mujeres 52. Edad media 57,4 $\pm 16,2$ (1887) años; SOFA $8,2 \pm 4,2$ (2-19) APACHE ॥ $19 \pm 8$ (3-40) SAPS \|I $48 \pm 20$ (11-95). La media de días de ingreso de los pacientes fue de 13,3 \pm 15 (2-139). Los pacientes requirieron oxigenoterapia de alto flujo en el contexto de insuficiencia respiratoria hipoxémica, en un $86 \%$ de los casos (110), hipercápnica en un $1 \%$ de los casos y mixta en un 13\% (17). Treinta y tres de los pacientes fueron clasificados como "grupo secundario" y 95 en el "grupo primario". Los días medios de uso de oxigenoterapia de alto flujo fue de $2,8 \pm$ $2,6(1-20)$. Un 50,8\% de los pacientes (65) requirieron ventilación mecánica invasiva. La mortalidad intra-UCI del estudio fue del 25,8\% (33). La mortalidad de los enfermos que requirieron intubación orotraqueal tras fracaso de oxigenoterapia de alto flujo, identificándose en las primeras 48 horas es del $44,8 \%$ en contra de pasadas estas 48 horas que es del $56,2 \%(p=0,3)$.

Los pacientes clasificados como "grupo secundario" (33) presentaron: Edad media de $59 \pm 15$ años, SOFA $10,3 \pm 3,9$; APACHE $\| 21,3 \pm 8$; SAPS $\| 55,2$ \pm 17 ; días de ingreso medio intra-UCI de $11,6 \pm 10$. Días de uso de oxigenoterapia de alto flujo: $2 \pm 1,4$. El uso de ventilación mecánica en este grupo fue del
$54 \%(18 / 33)$ y la mortalidad fue del $27,3 \%$.

Los pacientes clasificados en el "grupo primario" (95) presentaron: Edad media: 56,8 $\pm 16,6$ años; SOFA 7,5 \pm 4 ; APACHE $\| 18,3 \pm 7,8$; SAPS $\| 45 \pm 20$; días de ingreso intra-UCI $14 \pm 16,5$; Días de uso de oxigenoterapia de alto flujo: $3 \pm 3$. El uso de ventilación mecánica en este grupo fue del 49\% (47/95) y la mortalidad fue del 25,3\% (24/95).

Se objetivaron diferencias estadísticamente significativas en los valores de SOFA medio entre ambos grupos $(p<0,014)$ y APACHE II $(p<0,04)$. No se objetivaron diferencias en las variables edad media y días de ingreso en $\mathrm{UCl}$.

No se objetivaron diferencias estadísticamente significativas en el uso de ventilación mecánica entre el grupo clasificado como "grupo secundario" o "grupo primario" $(p=0,4)$. Tampoco se objetivó una mayor mortalidad en ninguno de los dos grupos ( $p=$ $0,5)$.

En el "grupo secundario", 18 pacientes requirieron intubación orotraqueal tras fracaso de la oxigenoterapia de alto flujo. En el $72,2 \%$ de los casos, el fallo fue identificado en las primeras 24 horas. En el grupo primario, hubo 47 pacientes que requirieron intubación orotraqueal. El fracaso fue identificado en el $76,59 \%$ de los casos en las primeras 24 horas. No existieron diferencias estadísticamente significativas $(p=0,5)$.

Aquellos pacientes que requirieron ventilación mecánica tras fracaso de oxigenoterapia de alto flujo recibieron una media de $2 \pm 1,7$ días oxigenoterapia de alto flujo previa a la intubación en el grupo de pacientes clasificados como "grupo primario". Recibieron una media de $2 \pm 1,9$ días oxigenoterapia de alto flujo en el grupo clasificado como "secundario" sin diferencias estadísticamente significativas $(p=0,9)$. (Tabla 1).

En el grupo clasificado como primario no se objetivó una mayor mortalidad, estadísticamente significativa, tras identificar el fracaso de la oxigenoterapia de alto flujo con más de 48 horas. $41,7 \%$ vs $63,6 \%$ $(p=0,17)$. Tampoco se objetivaron estas diferencias en el "grupo secundario". 54\% vs 40\% ( $p=0,5)$.

La media de oxigenoterapia de alto flujo previa a ventilación mecánica en los pacientes no supervivientes es de 2,36 \pm 2 días en el grupo de primario en contra de 1,9 \pm 2 días en el "grupo secudario" ( $p=$ $0,57)$. No existieron diferencias estadísticamente significativas en las medias de SOFA, APACHE II Y SAPS II entre los enfermos que fueron exitus en ambos grupos.

Las medias de los índices de gravedad fueron más altos, de forma estadísticamente significativa, 


\begin{tabular}{lccc}
\hline \multicolumn{2}{c}{ Tabla 1. Características de los pacientes en el grupo distress y neumonía } \\
\hline Causa insuficiencia respiratoria & Primaria & Secundaria & p \\
$\mathrm{n}$ & 95 & 33 & $\mathrm{p}=0,5$ \\
Edad media & 56,8 & 59 & $\mathrm{p}<0,014$ \\
SOFA & 7,5 & 10,3 & $<0,04$ \\
APACHE II & 18,3 & 21,3 & $\mathrm{p}<0,03$ \\
SAPS II & 45 & 55,2 & $\mathrm{p}=0,4$ \\
Días de ingreso & 14 & 11,6 & $\mathrm{p}=0,38$ \\
Ventilación mecánica & $49 \%$ & $54 \%$ & $\mathrm{p}=0,5$ \\
Mortalidad & $25,3 \%$ & $27,3 \%$ & $\mathrm{p}=0,077$ \\
Identificación de fallo primeras 48 & $76,6 \%$ & $72,2 \%$ & $\mathrm{p}=0,9$ \\
horas & & 2 & \\
$\begin{array}{l}\text { Días medios de OAF previo a la } \\
\text { intubación }\end{array}$ & 2 & $54 \%$ & \\
Mortalidad identificación de fallo & & $40 \%$ & \\
primeras 48 horas & $41,7 \%$ & & \\
Mortalidad identificación de fallo & & & \\
tras 48 horas & & & \\
\hline
\end{tabular}

en aquellos pacientes que requirieron una intubación orotraqueal y conexión a ventilación mecánica. Los enfermos clasificados como "exitus" también presentaron unos índices de gravedad más altos.

Se realizó un segundo análisis, dividiendo a los pacientes en: "Neumonía en pacientes inmunocompetentes" (57). "Neumonía en pacientes inmunodeprimidos" (38) y pacientes con "distress respiratorio de causa extrapulmonar" (33).

Las características de los pacientes inmunocompetentes con neumonía fueron: Edad media: 58,9 SOFA medio 6,8; APACHE ॥ 16,5; SAPS II: 41,6. Días de ingreso medio: 14; Días de uso de oxigenoterapia de alto flujo 2,8; uso de ventilación mecánica: 24/57 (42\%); mortalidad: 6/51 (10,5\%).

Las características de los pacientes inmunodeprimidos con neumonía fueron: Edad media: 53,7; SOFA 8,6; APACHE || 20,9; SAPS || 50,8. Días de ingreso medio intra-UCI: 13,7; días de uso de oxigenoterapia de alto flujo: 3,4; uso de ventilación mecánica: 23/38 (60,5\%); mortalidad 18/38 (47,4\%). Los pacientes clasificados como "inmunodeprimidos" presentaron como enfermedad de base: Leucemia linfoblástica aguda: 6 casos, leucemia mieloide aguda: 11, linfoma NK: 2, mieloma múltiple: 5, leucemia linfocítica crónica: 2, inmunodeficiencia común variable: 1 , linfoma de Hodking: 1, linfoma de No Hodking: 2, 7 pacientes se encontraban ingresados por estudio de pancitopinia o displasia, 1 paciente presentaba antecedentes de trasplante de médula como tratamiento de cáncer de testículo.

Las características de los pacientes con distress (33): Edad media: 56,8; SOFA 10,3 \pm 3,9; APACHE || 21,3 \pm 8; SAPS || 55,2 \pm 17 ; días de ingreso medio intra-UCl de 11,6 \pm 10 . Días de uso de oxigenoterapia de alto flujo: $2 \pm 1,4$. El uso de ventilación mecánica en este grupo fue del 54\% (18/33) y la mortalidad fue del 27,3\% (Tabla 2).

Al realizar la comparación entre el grupo de pacientes con neumonía, se objetivó que los pacientes inmunocompetentes presentan una mortalidad del $10,5 \%$ versus $47,4 \% p<0,001$; en los inmunodeprimidos con utilización de ventilación mecánica, en el $42 \%$ versus $60,5 \% p=0,06$. El tiempo medio del uso de oxigenoterapia de alto flujo previo a la ventilación mecánica invasiva fue 2,8 versus $3,4 p=0,4$. El tiempo medio de uso de la oxigenoterapia de alto flujo en los pacientes clasificados como "exitus" fue de 1,2 días en el "grupo de inmunocompetentes" y 2,6 en el "grupo inmunodeprimido" $p=0,5$.

En nuestra muestra, 65 pacientes fueron intubados tras el fracaso de la oxigenoterapia de alto flujo. De éstos, 49 fueron intubados en las primeras 48 horas, y 16 con más de 48 horas. En el primer grupo, se objetivó una mortalidad del $45 \%$ frente al $56 \%$ en el grupo con un retraso en la intubación mayor de 48 horas. Sin embargo, no se objetivaron diferencias estadísticamente significativas $(p=0,3)$. 


\begin{tabular}{lccc}
\hline \multicolumn{5}{c}{ Tabla 2. Características de los pacientes. Grupo Neumonia inmunocopetente, inmunodeprimido o } \\
distress
\end{tabular}

\begin{tabular}{lcc}
\multicolumn{2}{c}{ Tabla 3. Mortalidad tras fracaso de oxigenoterapia de alto flujo tras identificación en las primeras } \\
$\mathbf{4 8}$ horas o posteriores por grupo
\end{tabular}

En el grupo de pacientes "neumonía en inmunocompetentes", hubo 24 pacientes que requirieron una intubación orotraqueal tras fracaso de la oxigenoterapia de alto flujo; 20 fueron conectados a la ventilación mecánica en las primeras 48 horas, objetivándose una mortalidad del 15\%. En los pacientes conectados después de 48 horas (4) la mortalidad fue del $50 \%(p=0,18)$.

En el grupo de pacientes "neumonía en inmunodeprimido", hubo 23 pacientes que requirieron ventilación mecánica. Entre estos, hubo 16 pacientes conectados en las primeras 48 horas. La mortalidad objetivada fue del 75\%. Los pacientes conectados pasadas 48 horas, presentaron una mortalidad del $71 \%$ $(5 / 7)(p=0,6)$.

En el grupo de pacientes clasificados en el "grupo distress", hubo 18 pacientes que requirieron ventilación mecánica; 13 fueron conectados en las primeras 48 horas, objetivándose una mortalidad del 54\%. En los pacientes conectados tras 48 horas, se objetivó una mortalidad del 40\% $(p=0,5)$ (Tabla 3).

\section{Discusión}

La insuficiencia respiratoria es una causa de ingreso muy frecuente en las Unidades de cuidados Intensivos. La oxigenoterapia de alto flujo es una terapia de oxigenación reciente, sin embargo, su utilización es cada vez más frecuente en Unidades de Cuidados Intensivos[1] y Urgencias[16].

Una de las preguntas planteadas respecto a esta técnica es si evita o no el uso de ventilación mecánica. La ventilación mecánica es, por sí, un factor independiente de mortalidad en muchos de los grupos de enfermos ingresados en la UCI[17]. Por esta razón, evitar su uso puede reducir la mortalidad. Como respuesta a esta pregunta Frat JP et al, publicaron un estudio 
en la revista New England Journal of Medicine en la que comparaban esta técnica con oxigenoterapia estándar y ventilación mecánica no invasiva, concluyendo que la técnica evitaba intubaciones y disminuía la mortalidad[9].

Su indicación principal es la insuficiencia respiratoria hipoxémica. Muchos de los fracasos en el destete de la ventilación mecánica, se producen por esta causa. Para responder a la pregunta de si la oxigenoterapia de alto flujo puede evitar reintubaciones o facilitar la extubación programada, Hernández et al, publicaron en la revista JAMA un estudio para contestar a esta pregunta. En este, se concluye que el alto flujo facilita la extubación programada en pacientes de alto y bajo riesgo[10].

Otra pregunta planteada es si el retraso en intubación y conexión a ventilación mecánica, tras fracaso de la oxigenoterapia de alto flujo aumenta la mortalidad. Esta hipótesis se estudió en la ventilación mecánica no invasiva. La mayor parte de los estudios refieren que el retraso en la conexión a ventilación mecánica tras fracaso de ésta conlleva mayor mortalidad[18],[19]. Sin embargo, en la bibliografía también se refiere que el tiempo hasta la intubación en la ventilación mecánica no invasiva no aumenta la mortalidad[20]. En el caso de la oxigenoterapia de alto flujo, hay varios estudios que indican que el retraso en la intubación tras su fracaso, conlleva mayor mortalidad[11].

En nuestro estudio, el 50,8\% (65) de los pacientes requirieron intubación y conexión a ventilación mecánica tras fracaso de la oxigenoterapia de alto flujo, según los criterios mencionados. De estos, en el 75,4\% de los casos, el fracaso se objetivó en las primeras 48 horas, mientras que en el $24,6 \%$ de los casos fueron intubados con más de 48 horas de terapia. Sin embargo, pese a que el porcentaje de mortalidad es mayor en el segundo grupo (44,8\% versus $56,2 \%)$, no objetivamos diferencias estadísticamente significativas en cuanto a mortalidad $(p=0,3)$.

Otra de las preguntas que intentamos responder en este estudio es si los pacientes presentaban distinta respuesta a la oxigenoterapia de alto flujo según su patología de base. Los pacientes que requieren oxigenoterapia de alto flujo por insuficiencia respiratoria, cuya etiología es neumonía en inmunocompetentes, requirieron ventilación mecánica en un $42 \%$ de los casos en contra del $60,5 \%$ en pacientes con neumonía en inmunodeprimido y $54 \%$ en el "grupo distress" causa extrapulmonar. Sin embargo, pese a que los porcentajes de necesidad de ventilación mecánica son mayores en el caso de neumonía en inmunodeprimido, no se alcanza la significación estadística con respecto a los otros grupos. El fracaso de la oxigenoterapia de alto flujo no se asocia de forma estadísticamente significativa a la patología subyacente. Sí se objetivan diferencias en cuanto a mortalidad global por grupos.

En cuanto a la mortalidad según retraso en la intubación tras fracaso de oxigenoterapia de alto flujo, tampoco se objetivaron diferencias por grupo de enfermos en cuanto a la identificación del fracaso en las primeras 48 horas. Sí se objetivan diferencias estadísticamente significativas en cuanto a mortalidad de los pacientes intubados en las primeras 48 horas tras el fracaso de oxigenoterapia de alto flujo. Los pacientes con neumonía en inmunocompetentes presentaron una mortalidad del $15 \%$ versus $75 \%$, y $54 \%$ en los grupos neumonía en inmunocomprometido y distress extrapulmonar. Estas diferencias no se aprecian al analizar la mortalidad en los diferentes grupos tras conexión a ventilación mecánica pasadas 48 horas: Mortalidad del $50 \%$ en el grupo de "neumonía en inmunocompetente" versus $71 \%$ en el grupo de "neumonía en inmunocomprometido" y $40 \%$ en el "grupo distress".

Con los resultados obtenidos, podemos concluir que la oxigenoterapia de alto flujo es una opción segura en el tratamiento de la insuficiencia respiratoria aguda. El fracaso de esta terapia no depende de la patología de base en nuestro estudio. Sin embargo, la necesidad de ventilación mecánica y mortalidad sí se asocian con los índices de gravedad.

En nuestro estudio, el retraso en la intubación orotraqueal tras fracaso de la oxigenoterapia de alto flujo, no se asocia de forma estadísticamente significativa con la mortalidad, aunque ésta es superior en el grupo cuyo fracaso se identifica con más de 48 horas. El punto más destacable en cuanto al retraso por grupo de enfermo, es el observado en la mortalidad de los pacientes inmunocompetentes con neumonía. Éstos presentan una menor mortalidad que los otros grupos, esta mortalidad menor, se objetiva cuando el paciente es intubado en las primeras 48 horas. Pasadas estas 48 horas, la mortalidad se iguala con los otros grupos. Nuestra explicación a este suceso es que la insuficiencia respiratoria, cuya etiología es la neumonía en inmunocompetente, presenta un mejor pronóstico que en el paciente inmunodeprimido o distress extrapulmonar. Esta diferencia se iguala ante el retraso de la intubación orotraqueal tras fracaso de la terapia de alto flujo.

\section{Conclusiones}

Los resultados de la oxigenoterapia de alto flujo no parecen ser diferentes en la insuficiencia respira- 
toria de causa pulmonar o extrapulmonar tanto en el porcentaje de fracasos o mortalidad.

La gravedad del enfermo se relaciona con la necesidad de ventilación mecánica y mortalidad. Sin embargo, el paciente que más parece beneficiarse de la rápida identificación del fracaso de la oxigenoterapia de alto flujo es el paciente con neumonía inmuno- competente.

El paciente con insuficiencia respiratoria, cuya etiología es neumonía en inmunocompetente presenta un menor porcentaje de mortalidad. Esta mortalidad se iguala con otros grupos cuando se identifica el fracaso de la oxigenoterapia de alto flujo con más de 48 horas de tratamiento.

\section{Referencias}

1. Demoule A, Girou E, Richard JC, Taillé S, Brochard L. Increased use of noninvasive ventilation in French intensive care units. Intensive Care Med. 2006 Nov;32(11):1747-55.

2. Cuquemelle E, Pham T, Papon $J F$, Louis B, Danin PE, Brochard L. Heated and humidified highflow oxygen therapy reduces discomfort during hypoxemic respiratory failure. Respir Care. 2012 Oct;57(10):1571-7.

3. Sztrymf B, Messika J, Bertrand $F$, Hurel D, Leon R, Dreyfuss D, et al. Beneficial effects of humidified high flow nasal oxygen in critical care patients: a prospective pilot study. Intensive Care Med. 2011 Nov;37(11):1780-6.

4. Parke RL, McGuinness SP. Pressures delivered by nasal high flow oxygen during all phases of the respiratory cycle. Respir Care. 2013 Oct;58(10):1621-4.

5. Groves N, Tobin A. High flow nasal oxygen generates positive airway pressure in adult volunteers. Aust Crit Care. 2007 Nov;20(4):126-31.

6. Parke RL, Eccleston ML, McGuinness SP. The effects of flow on airway pressure during nasal high-flow oxygen therapy. Respir Care. 2011 Aug;56(8):1151-5.

7. Bazuaye EA, Stone TN, Corris PA, Gibson GJ. Variability of inspired oxygen concentration with nasal cannulas. Thorax. 1992 Aug;47(8):609-11.
8. Nedel WL, Deutschendorf C, Moraes Rodrigues Filho E. HighFlow Nasal Cannula in Critically III Subjects With or at Risk for Respiratory Failure: A Systematic Review and Meta-Analysis. Respiratory Care [Internet]. Daedalus Enterprises; 2016 Nov 22;62(1):123-32.

9. Frat JP, Thille AW, Mercat A, Girault $C$, Ragot $S$, Perbet $S$, et al.; FLORALI Study Group; REVA Network. High-flow oxygen through nasal cannula in acute hypoxemic respiratory failure. N Engl J Med. 2015 Jun;372(23):2185-96.

10. Hernández G, Vaquero Collado C, García Plaza S, Villasclaras Pacheco A, Pardo Rey C, de la Fuente $\mathrm{O}^{\prime}$ Connor $\mathrm{E}$, et al. High Flow Conditioned Oxygen Therapy for Prevention of Reintubation in Critically III Patients: A Preliminary Cohort Study. Int J Crit Care Emerg Med. 2015;1(2):2.

11. Kang BJ, Koh $Y$, Lim CM, Huh JW, Baek S, Han M, et al. Failure of high-flow nasal cannula therapy may delay intubation and increase mortality. Intensive Care Med. 2015 Apr;41(4):623-32.

12. Roca O, Messika J, Caralt B, García-de-Acilu M, Sztrymf B, Ricard $J D$, et al. Predicting success of high-flow nasal cannula in pneumonia patients with hypoxemic respiratory failure: the utility of the ROX index. J Crit Care. 2016 Oct;35:200-5.

13. Lemiale $V$, Mokart $D$, RescheRigon M, Pène F, Mayaux J, Faucher E, et al.; Groupe de
Recherche en Réanimation Respiratoire du patient d'Onco-Hématologie (GRRR-OH). Effect of noninvasive ventilation vs oxygen therapy on mortality among immunocompromised patients with acute respiratory failure: a randomized clinical trial. JAMA. 2015 Oct;314(16):1711-9.

14. Epstein AS, Hartridge-Lambert SK, Ramaker JS, Voigt LP, Portlock CS. Humidified high-flow nasal oxygen utilization in patients with cancer at Memorial Sloan-Kettering Cancer Center. J Palliat Med. 2011 Jul;14(7):8359.

15. Hui D, Morgado M, Chisholm G, Withers L, Nguyen Q, Finch C, et al. High-flow oxygen and bilevel positive airway pressure for persistent dyspnea in patients with advanced cancer: a phase II randomized trial. J Pain Symptom Manage. 2013 Oct;46(4):46373.

16. Jones PG, Kamona S, Doran O, Sawtell F, Wilsher M. Randomized controlled trial of humidified high-flow nasal oxygen for acute respiratory distress in the emergency department: the HOT-ER study. Respir Care. 2016 Mar;61(3):291-9.

17. Bickenbach J, Fries $M, \operatorname{Rex} S$, Stitz C, Heussen N, Rossaint R, et al. Outcome and mortality risk factors in long-term treated ICU patients: a retrospective analysis. Minerva Anestesiol. 2011 Apr;77(4):427-38.

18. Mosier JM, Sakles JC, Whit- 
more SP, Hypes CD, Hallett DK, Hawbaker KE, et al. Failed noninvasive positive-pressure ventilation is associated with an increased risk of intubation-related complications. Ann Intensive Care. 2015 Mar;5(1):4.

19. Chandra D, Stamm JA, Taylor
B, Ramos RM, Satterwhite L, Krishnan JA, et al. Outcomes of noninvasive ventilation for acute exacerbations of chronic obstructive pulmonary disease in the United States, 1998-2008.

Am J Respir Crit Care Med. 2012 Jan;185(2):152-9.
20. Arnaud W. ThilleEmail author, Damien Contou, Chiara Fragnoli, Ana Córdoba-Izquierdo, Florence Boissier and Christian BrunBuisson. Non-invasive ventilation for acute hypoxemic respiratory failure: intubation rate and risk factors. Crit Care. 2013;17(6):1. 